\title{
Successful Anesthetic Management for Surgical Repair of Atrio-Esophageal Fistula Following Radiofrequency Ablation for Atrial Fibrillation
}

\author{
Atsushi Yasuda, Paul H. Alfille, Lisa T. Wollman-Kliman \\ Department of Anesthesia, Critical Care and Pain Medicine, Massachusetts General Hospital, Boston, USA. \\ Email: ayasuda@partners.org \\ Received July $17^{\text {th }}, 2013$; revised August $15^{\text {th }}, 2013$; accepted August $26^{\text {th }}, 2013$ \\ Copyright (C) 2013 Atsushi Yasuda et al. This is an open access article distributed under the Creative Commons Attribution License, \\ which permits unrestricted use, distribution, and reproduction in any medium, provided the original work is properly cited.
}

\begin{abstract}
Introduction: Atrio-esophageal fistula is a rare but often fatal complication of radiofrequency ablation for atrial fibrillation. Here we report a successful case in anesthetic management of surgical repair of atrio-esophageal fistula. Case Report: The patient was a 56-year-old man status post radiofrequency ablation for atrial fibrillation one month before presenting with fever and symptoms and signs of cerebral emboli. He was diagnosed as having atrio-esophageal fistula, which required emergent surgical repair. In the operating room, rapid sequence induction was performed with avoidance of positive pressure ventilation before securing airway. Double lumen tube was used for lung isolation for left thoracotomy. Upon exploration, a small fistula was identified. Both atrial and esophageal defects were ligated and an intercostal muscle flap was placed. The patient's heart rhythm was atrial flutter/atrial fibrillation with marginal hemodynamics during the procedure, but cardioversion was delayed until the fistula was repaired and no remaining air, blood clot or gastric content in the heart was confirmed by epicardial ultrasound. The patient tolerated the surgery and was transferred to ICU, intubated and ventilated. He recovered from surgery and was transferred to a rehabilitation hospital with residual expressive aphasia. Conclusion: We had a successful case in anesthetic management for surgical repair of atrio-esophageal fistula by preventing massive bleeding as well as multiple air embolization through the fistula.
\end{abstract}

Keywords: Atrial Fibrillation; Radiofrequency Ablation; Complication; Atrio-Esophageal Fistula; Anesthetic Management

\section{Introduction}

Atrial fibrillation is the most common clinically relevant heart rhythm disorder. Radiofrequency ablation is widely performed as a treatment for recurrent, drug-resistant atrial fibrillation. Atrio-esophageal fistula is a rare but often fatal complication of radiofrequency ablation for atrial fibrillation. There are no reports on anesthetic management for surgical repair of atrio-esophageal fistula. Here we report a successful case in anesthetic management of 56-year-old man who underwent emergent surgical repair of atrio-esophageal fistula.

\section{Case Report}

The patient was a 56-year-old man (85 kgw) with history of chronic deep venous thromboses (DVT) and factor $\mathrm{V}$ Leiden deficiency on warfarin therapy and recurrent paroxysmal atrial fibrillation. He underwent successful ra- diofrequency ablation/pulmonary vein isolation for atrial fibrillation at an outside institution. The initial post-procedure course was uneventful and he maintained normal sinus rhythm. One month later he developed symptoms of progressive malaise, high fevers, and intermittent speech difficulty, which required hospitalization to the outside hospital. Blood cultures drawn at presentation later grew streptococcus viridans. Transesophageal echocardiogram was negative for endocarditis but revealed a small patent foramen ovale. Lower extremity non-invasive sonography showed bilateral DVTs. MRI of the brain showed multiple subacute embolic foci. He was transferred to our tertiary hospital 3 days after the initial admission.

On arrival to our institution, CT scan of the chest was performed, showing air pockets in the left atrial appendage, left ventricle, and left superior pulmonary vein (Figure 1). A gastrografin swallow test showed extravasation 


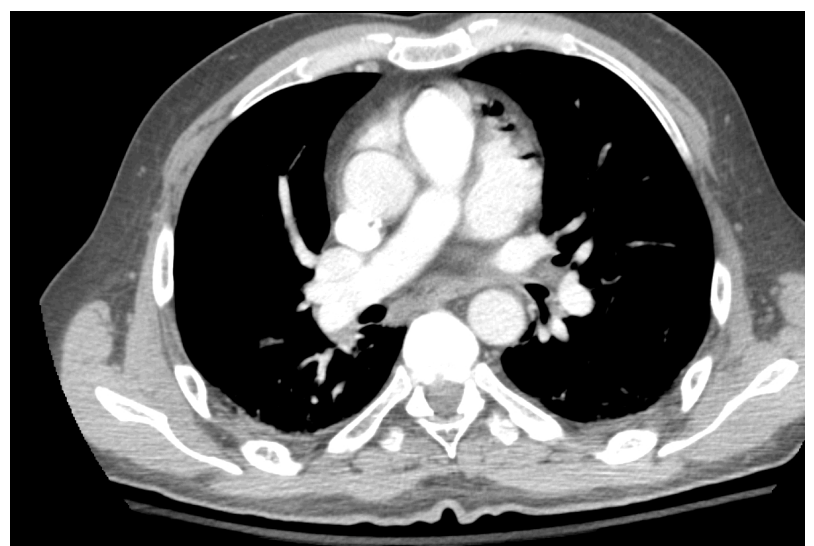

Figure 1. CT scan of the chest on arrival to our institution showed air pockets in the left atrial appendage, left ventricle, and left superior pulmonary vein.

of the contrast from the mid-esophagus with rapid washout. The patient experienced a progressive neurologic decline, became somnolent and was emergently taken to the operating room (OR) with a diagnosis of atrio-esophageal fistula on hospital day 2.

In the OR, we placed two 14 gage peripheral intravenous lines, a 9 French introducer in right internal jugular vein and a 20 gage left radial arterial line prior to the induction of anesthesia. Cardiopulmonary bypass equipment was brought to the OR on standby. The patient's heart rhythm was found to be atrial flutter/atrial fibrillation with marginal hemodynamics (heart rate 140 to 160 , blood pressure 100 - 120/60 - $80 \mathrm{mmHg}$ on norepinephrine infusion up to $5 \mathrm{mcg} / \mathrm{min}$ from arrival to the OR throughout the procedure). The patient was preoxygenated with $100 \%$ oxygen and was induced with intravenous $50 \mathrm{mg}$ of ketamine, $250 \mathrm{mcg}$ of fentanyl and $20 \mathrm{mg}$ of cisatracurium. Cricoid pressure was applied. An oral cuffed 8.0 mmID endotracheal tube was inserted. Bronchoscopy was performed to check whether the tracheal and bronchial walls were intact, followed by replacement of endotracheal tube with 39Fr right side double lumen tube (DLT). The tube position was confirmed by flex bronchoscopy. The patient was placed in the right lateral decbitus position as surgical exposure was via a left thoracotomy.

Upon exploration, no pleural effusion was noted. There was inflammatory adherence of the esophagus just superior to the inferior pulmonary vein. There was a tiny contained mediastinal abscess between the wall of the esophagus and the posterior left atrium. A small fistula was identified. Both atrial and esophageal defects were ligated and an intercostal muscle flap was placed between the atrial and esophageal repair. Once the surgeon were satisfied with the repair, epicardial ultrasound was performed to assess whether there was any air, blood clot or gastric content in the heart prior to cardioversion; there were no such findings. It was then decided to apply 5 Joules direct synchronous cardioversion, which was delivered through internal paddles. The rhythm immediately converted to sinus rhythm. After the surgery completed, the patient was turned supine and DLT was changed back to a single lumen endotracheal tube. The patient was transferred to ICU, intubated, and ventilated primarily as a result of the patient's depressed mental status.

In the ICU the patient was treated with intravenous antibiotics and total parenteral nutrition. The patient recovered slowly, was extubated on ICU day 2, was discharged from ICU on ICU day 6 and was transferred to a rehabilitation hospital. His neurologic status improved slowly. Although he continued to suffer expressive aphasia, he was able to be independent with his activities of daily living on discharge from the rehabilitation hospital 45 days after the surgery.

\section{Discussion}

Radiofrequency ablation for the treatment of atrial fibrillation is becoming more widely practiced in the United States. Cases of atrio-esophageal fistula, a rare but usually fatal complication of this procedure have been reported [1-10]. This is the first report of detailed anesthetic management for surgical repair of atrio-esophageal fistula. We report our successful management and the anesthetic considerations.

The use of cardiopulmonary bypass for repair of atrioesophageal fistula has been described $[4,5]$. There is probably increased risk of embolization and bleeding when cardiopulmonary bypass is used [4]. We had cardiopulmonary bypass standby in the event cardiac standstill was required for surgical repair. But, the atrio-esophageal fistula was small and confined, and the surgical repair was successful without cardiopulmonary bypass in our case.

Transesophageal echocardiography (TEE) is commonly used in cardiac surgery. The decision not to perform a TEE was made to avoid creating a larger defect in the esophagus and/or causing bleeding in light of the diagnosis.

Induction was performed with ketamine to maintain hemodynamics, keeping the patient's spontaneous breathing and thereby to avoid positive pressure ventilation before airway was secured. Positive pressure ventilation before securing airway would likely cause air embolization from the esophagus to the left atrium through the fistula. Once airway was secured, positive pressure ventilation was carefully applied.

The biggest discussion between the surgeons and the anesthesiologists was when to convert hemodynamically 
unstable atrial fibrillation to sinus rhythm. The patient became hemodynamically unstable shortly after arrival to the OR. According to ACLS protocol, hemodynamically unstable atrial fibrillation should be cardioverted. But in this specific case, atrial fibrillation kept atrial pressure low enough to avoid hemorrhage from the atria to the chest or esophagus. Also converting to sinus rhythm increases the likelihood of embolization of air, blood clot, gastric contents in the left atrium into the brain or other organs. And also the patient had had atrial fibrillation for at least several days and the patient had very high risk for thrombus formation due to factor $\mathrm{V}$ Leiden deficiency and DVT. We did not know whether there was thrombus or air in the left atria or how big it would be if thrombus was present at this point. Cardioversion likely could have ended up causing massive bleeding or embolization. Hemodynamics was managed instead by infusing phenylephrine and norepinephrine to maintain a systolic blood pressure around $100 \mathrm{mmHg}$. Only once air in the atria was suctioned, and no other air, blood clots or gastric contents were detected by direct epicardial ultrasound and the fistula was surgically repaired, caridoversion was performed by directly applying paddles to the heart.

The patient was kept intubated and transferred to the ICU due to the hemodynamic instability and neurological deterioration shown preoperatively which could impair airway protection postoperatively. The patient was treated for sepsis with broad spectrum of antibiotics initially to cover the likely bacterial species inhabiting esophagus.

The role of esophageal stent was considered intraoperatively. There was a successful case of esophageal stent for atrio-esophageal fistula, using esophagoscopy [7], even though most of the literature did recommend avoidance of esophagoscopy [4-6,9]. But more data is needed to determine which cases are indicated for esophageal stent and esophagoscopy.

The key to managing these patients' safety is the prevention of massive bleeding and multiple air embolization during the surgical repair and then later the postoperative management of sepsis and multiple organ failure, which are the main causes of death from this complication.

\section{Conclusion}

We had a successful case in anesthetic management for surgical repair of atrio-esophageal fistula with avoidance of positive pressure ventilation before securing airway and careful control of hemodynamics by delaying cardioversion to prevent massive bleeding as well as multiple air embolization through the fistula.

\section{REFERENCES}

[1] A. M. Gillinov, G. Pettersson and T. W. Rice, "Esophag- eal Injury during Radiofrequency Ablation for Atrial Fibrillation," The Journal of Thoracic and Cardiovascular Surgery, Vol. 122, No. 6, 2001, pp. 1239-1240. http://dx.doi.org/10.1067/mtc.2001.118041

[2] N. Doll, M. A. Borger, A. Fabricius, S. Stephan, J. Gummert, F. W. Mohr, et al., "Esophageal Perforation during Left Atrial Radiofrequency Ablation: Is the Risk Too High?” The Journal of Thoracic and Cardiovascular Surgery, Vol. 125, No. 4, 2003, pp. 836-842. http://dx.doi.org/10.1067/mtc.2003.165

[3] G. Hindricks and H. Kottkamp, "Potential Benefits, Risks, and Complications of Catheter Ablation of Atrial Fibrillation: More Questions Than Answers,” Journal of Cardiovascular Electrophysiology, Vol. 13, No. 8, 2002, pp. 768769. http://dx.doi.org/10.1046/j.1540-8167.2002.00768.x

[4] B. Sonmez, E. Demirsoy, N. Yagan, M. Unal, H. Arbatli, D. Sener, et al., "A Fatal Complication Due to Radiofrequency Ablation for Atrial Fibrillation: Atrio-Esophageal Fistula,” The Annals of Thoracic Surgery, Vol. 76, No. 1, 2003, pp. 281-283.

http://dx.doi.org/10.1016/S0003-4975(03)00006-7

[5] C. Pappone, H. Oral, V. Santinelli, G. Vicedomini, C. C. Lang, F. Manguso, et al., "Atrio-Esophageal Fistula as a Complication of Percutaneous Transcatheter Ablation of Atrial Fibrillation,” Circulation, Vol. 109, No. 22, 2004, pp. 2724-2726.

http://dx.doi.org/10.1161/01.CIR.0000131866.44650.46

[6] M. I. Scanavacca, A. D’avila, J. Parga and E. Sosa, “Left Atrial-Esophageal Fistula Following Radiofrequency Catheter Ablation of Atrial Fibrillation," Journal of Cardiovascular Electrophysiology, Vol. 15, No. 8, 2004, pp. 960962. http://dx.doi.org/10.1046/j.1540-8167.2004.04083.x

[7] T. J. Bunch, J. Nelson, T. Foley, S. Allison, B. G. Crandall, J. S. Osborn, et al., “Temporary Esophageal Stenting Allows Healing of Esophageal Perforations Following Atrial Fibrillation Ablation Procedures," Journal of Cardiovascular Electrophysiology, Vol. 17, No. 4, 2006, pp. 435-439.

http://dx.doi.org/10.1111/j.1540-8167.2006.00464.x

[8] P. Schley, H. Gu“lker and M. Horlitz, “Atrio-Oesophageal Fistula Following Circumferential Pulmonary Vein Ablation: Verification of Diagnosis with Multislice Computed Tomography,” Europace, Vol. 8, No. 3, 2006, pp. 189190. http://dx.doi.org/10.1093/europace/euj050

[9] J. E. Cummings, R. A. Schweikert, W. I. Saliba, J. D. Burkhardt, F. Kilikaslan, E. Saad, et al., "Brief Communication: Atrial-Esophageal Fistulas after Radiofrequency Ablation," Annals of Internal Medicine, Vol. 144, No. 8, 2006, pp. 572-574.

http://dx.doi.org/10.7326/0003-4819-144-8-200604180-0 0007

[10] A. Takahashi, T. Kuwahara and Y. Takahashi, "Complications in the Catheter Ablation of Atrial Fibrillation: Incidence and Management," Circulation Journal, Vol. 73, No. 2, 2009, pp. 221-226. http://dx.doi.org/10.1253/circj.CJ-08-1097. 